\title{
A Study of High-Precision Time-Synchronization for TDoA-Based Location Estimation
}

\author{
Kim Jae Wan ${ }^{\dagger} \cdot$ Eom Doo Seop ${ }^{++}$
}

\begin{abstract}
Presently, there are many different technologies used for position detection. However, as signal-receiving devices operating in different locations must detect the precise position of objects located at long distances, it is essential to know the precise time at which an object's or a user's terminal device sends a signal. For this purpose, the existing time of arrival (ToA) technology is not sufficiently reliable, and the existing time difference of arrival (TDoA) technology is more suitable. If a TDoA-based electric surveillance system and other tracking devices fail to achieve precise time-synchronization between devices with separation distance operation, it is impossible to obtain correct TDoA values from the signals sent by the signal-receiving devices; this failure to obtain the correct values directly affects the location estimation error. For this reason, the technology for achieving precise time synchronization between signal-receiving devices in separation distance operation, among the technologies previously mentioned, is a core technology for detecting TDoA-based locations. In this paper, the accuracy of the proposed time synchronization and the measurement error in the TDoA-based location detection technology is evaluated. The TDoA-based location measurement error is significantly improved when using the proposed method for time-synchronization error reduction.
\end{abstract}

Keywords : Localization, Time-Synchronization, TDoA, TPSN, Time Stamping

\section{$\mathrm{TDoA}$ 기반의 위치 추정을 위한 초정밀 시각동기에 관한 연구}

\author{
김 재 완 ${ }^{+}$엄 두 섭 ${ }^{++}$
}

요 약

현재 위치인식에 관한 많은 기술들이 존재한다. 하지만 서로 다른 곳에 위치한 신호수신장치들이 먼 곳에 위치한 특정 물체의 위치를 정확 하게 알아내기 위해서는 해당 물체 또는 사용자의 단말장치에서 내보내는 신호의 정확한 시간을 알아야 한다. 이러한 목적 때문에 ToA 기술보 다는 $\mathrm{TDoA}$ 기술이 보다 적합하다. TDoA 기술을 기본으로 하는 전자 감시 시스템 및 다른 위치추적 장치들은 이격 장치들간의 정밀한 시각동 기가 되지 않아서 신호수신장치들에 의해 보내지는 신호로부터 정확한 $\mathrm{TDoA}$ 값을 획득하는 것이 불가능하다. 이렇게 직접적으로 정확한 값을 받지 못함으로써 위치 추정 에러가 발생된다. 이러한 이유로 이격 장치들간의 정밀한 시각동기 기술은 TDoA를 기반으로 하는 위치인식에 있 어서 필수적인 것이다. 본 논문에서는 $\mathrm{TDoA}$ 를 기반으로 하는 위치인식을 위해, 제안된 정밀한 시각동기 기술의 정확성과 측정오차가 평가된 다. TDoA를 기반으로 하는 위치 측정 오차는 제안하는 시각동기 알고리즘을 사용할 때 크게 향상된다.

키워드 : 위치인식, 시각동기, TDoA, TPSN, 타임 스탬핑

\section{Introduction}

As shown below in Fig. 1, the TDoA-based electronic position detection system is composed of signal reception devices isolated by space (a minimum of 4 is needed for

\footnotetext{
† 준 회 원 : 고려대학교 전자컴퓨터공학과 박사과정

††비 회 원: 고려대학교 전자컴퓨터공학과 정교수

논문접수: 2012년 9월 26일

수 정 일 : 1 차 2012 년 12 월 3 일

심사완료 : 2012년 12월 12일

* Corresponding Author : Eom Doo Seop(eomds@korea.ac.kr)
}

3-dimensional location determination), a center control system for location calculation, and wireless communication equipment for communication between them.

To develop a TDoA-based electric surveillance system that detects long-distance threats and performs threat location in real time, each of the following is required: (1) high-sensitivity digital signal receiver technology; (2) technology for precise time synchronization between signal receiving devices for separation distance operation 


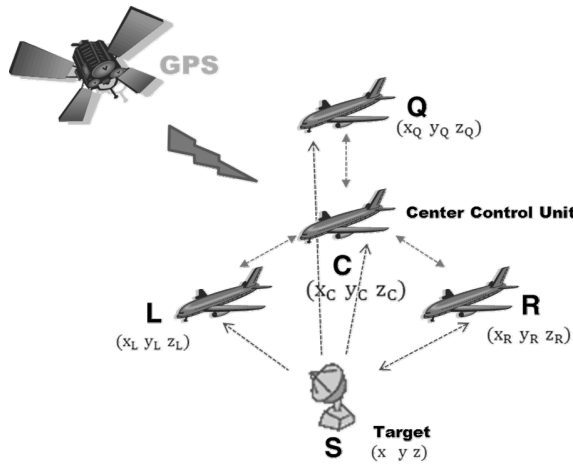

Fig. 1. TDoA-based position detection system

[1-5]; and (3) real-time technology to estimate and trace the threat location with the use of TDoA [6, 7]. If a TDoA-based electric surveillance system fails to achieve precise time synchronization between devices in separation distance operation, it is impossible to obtain correct TDoA values from signals sent by signal-receiving devices; this failure to obtain the values directly affects the location estimation error.

The synchronization method in the proposed system uses the global positioning system (GPS) [8] so as to overcome the performance limitations of the common-view GPS synchronization system, which is a two-way communication system. Further, using the time-synchronization protocol to exchange time-stamp message reduces the burden of hardware realization in the two-way communication link construction and ensures the flexibility of the system.

The proposed system uses a highly accurate OCXO, hardware design to improve the oscillator's accuracy, using the GPS timing signal, time difference estimation using the two-way communication link, and time-synchronization protocol. The system applies a compensation algorithm to correct the synchronization error in the receiver's clock offset and clock skew and thereby improves the location detection error when using the TDoA by reducing the time-synchronization error to less than $5 \mathrm{~ns}$.

\section{Related Works}

Traditional time-synchronization techniques based on wired networks are difficult to apply to the wireless network environment. The problems that arise are associated with low-power operation, mobility, and network automatic formation. For time synchronization in the wireless network environment, the following items should be considered:
- Other resources to process communications, energy, and the wireless network

- Expansion of the wireless network

- Time-synchronization infrastructure for the wireless network

- The node set of the wireless network

\subsection{TPSN}

TPSN is one of the time--synchronization protocols that is suitable for the wireless network environment. It begins at the root node for time synchronization and forms a hierarchical structure network with different levels. To check the synchronization between nodes, exchanges time-stamp messages, and creates higher-level nodes, it calculates the offset and delivery delay and corrects the angle.

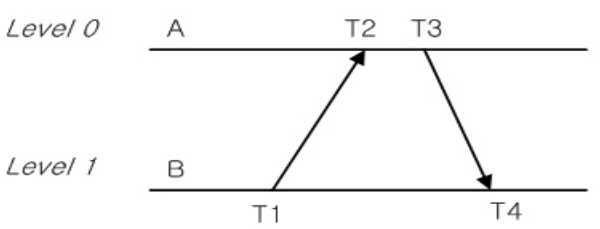

Fig. 2. Two-step message exchange in TPSN

Fig. 2 shows the method of synchronization of sensor $\mathrm{B}$ to sensor node A's timer through a two-step message exchange on the TPSN. T1 shows sensor node B's transmission time, T2 is sensor node A's reception time for the message, T3 is sensor node A's transmission time for the Ack message, and T4 is sensor node B's reception time for information.

When the two-step message exchange has been completed, sensor node B can look for the timer offset $(\Delta)$ and EM wave delay time (d) using the following formula, as in (1), and, when correcting by adding $\Delta$ to the timer value, sensor A's timer and the synchronization can be maintained.

$$
\begin{aligned}
& \Delta=\frac{\left(T_{2}-T_{1}\right)-\left(T_{4}-T_{3}\right)}{2} \\
& d=\frac{\left(T_{2}-T_{1}\right)+\left(T_{4}-T_{3}\right)}{2}
\end{aligned}
$$

\subsection{Time-Synchronization Error in Wireless Network}

Fig. 3 shows a model of the packet delay that occurs in the course of exchanging the packet (for time-stamp message transmission) for two receivers on the wireless link. The packet delay can be classified into three types of delays: delay in the transmission process, EM wave delay (the process of delivery via the wireless link), and delay in the reception process. 


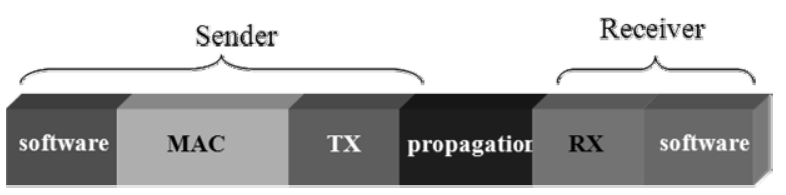

Fig. 3. Delay model for processing of time-stamp message transmission

The delay in the transmission process is referred to as "send time" occurring at the time-synchronization application software, the "needed-access" time accessing the wireless channel in the MAC layer, and the "needed-transmission" time delivering the packet bitwise in the physical layer. Moreover, the delay of the reception course is composed of the receive time needed to deliver the time-stamp message to the time-synchronization application (in the application layer) and the reception time needed to reach the MAC layer by receiving the bit stream (from the physical layer). The timesynchronization error is generated at the physical clock [9-12]. The CPU uses a crystal oscillator as its external clock. In the case of the correction radiator, error occurs in the clock of the applicable frequency, but through aging, temperature, or humidity, and, in the case of a supply voltage change, it results in a clock with a frequency that is slightly skewed from the actual frequency. Because of this clock skew, although the synchronization between receivers is performed at a particular moment, the two-receiver clock time becomes more distant as time passes by. Therefore, in the case of special purposes (such as long-term or electronic operation), a fuller analysis of the time-synchronization process is needed, and the algorithm development must achieve accurate synchronization.

\section{Proposed System}

For time-synchronizations with less than 5 ns synchronization error between the isolated operation signal receivers of a TDoA-based electronic surveillance system, the goal is precise time synchronization using GPS clock synchronization and a two-way data-link-based clock error correction.

The proposed system's approximate structure is shown in Fig. 4.

\subsection{Clock Generator}

The clock generator used in the master and slave node is composed of a GPS receiver, a DPLL, an OCXO, a

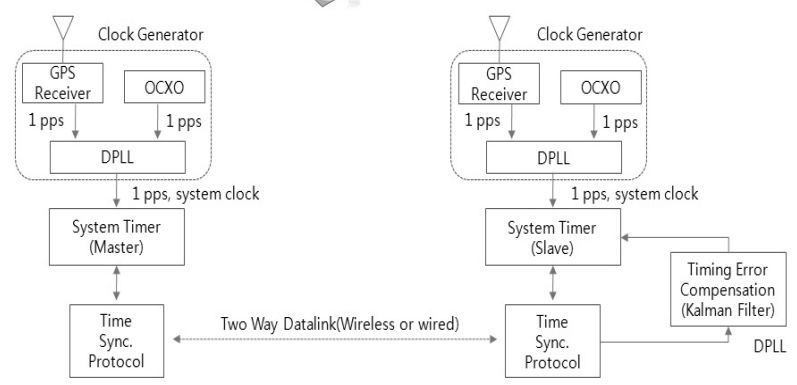

Fig. 4. Proposed time-synchronization system

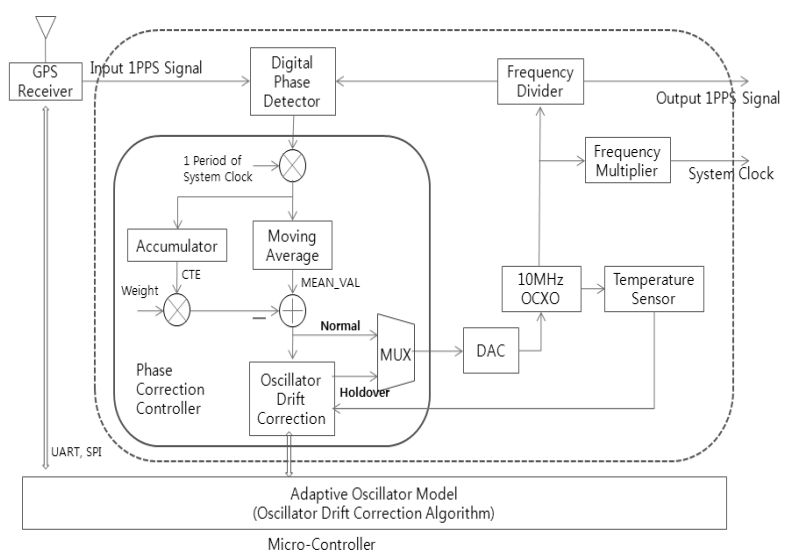

Fig. 5. Clock generator

temperature sensor, and an oscillator drift compensation algorithm, as shown in Fig. 5.

The DPLL is used to perform synchronization in the OXCO with a 1 PPS signal, providing synchronization with the GPS receiver and forming the system clock (1 $\mathrm{GHz}$ ), which has a high frequency stability of approximately $0.001 \mathrm{ppb}$ (parts per billion). The 1 PPS signal of the GPS receiver inputting to the DPLL has a short-term jitter of 30 ns through the effect of reflection and EM wave speed change in the ionization layer, multipath reception, and others.

Because the DPLL generates a signal using the GPS satellite's atomic clock (which has high stability), it can reduce the jitter to $0.3 \mathrm{~ns}$ in the case of filtering through the loop filter in the DPLL. In the case of the GPS signal that is not received, it uses the holdover mode, which can maintain a high level of frequency stability. The holdover mode uses the DPLL and Kalman filter of the frequency change characters following the OCXO's temperature and time to synchronize the GPS's timing signal. By modeling adaptively, the OCXO's high level of frequency stability can be maintained in the condition in which it is not being synchronized to the GPS's timing signal. 


\subsection{Two-Way Communication Link}

As mentioned previously, in the proposed timesynchronization system, to achieve accurate time synchronization between the isolated application signal receiver, the system uses a two-way communication link, which is known to be the best method. In this method, the system uses the time-stamp message on the two-way communication link between the master and the slave node, and the delays are mutually offset when the two-way communication link is the same throughout the delivery of the clock phase. Therefore, clock difference estimation can be performed for two receivers. To make the two-way delay of the time-stamp message the same, it is necessary that the delay change be prevented by performing all processing using hardware. Furthermore, it is better to perform time-stamping in the nearest part of the transmission medium connected to the node's physical layer.

As shown in Fig. 6, when using time-stamping in the physical layer, the uncertainty factor in the transmission and reception course is the propagation time. When the master and slave nodes have a short baseline and LOS is secured, the two-way electric wave delay difference is small, and therefore, accurate estimation of clock difference between the two nodes is possible. For this reason, the proposed time--synchronization system performs time-stamping in the physical layer.

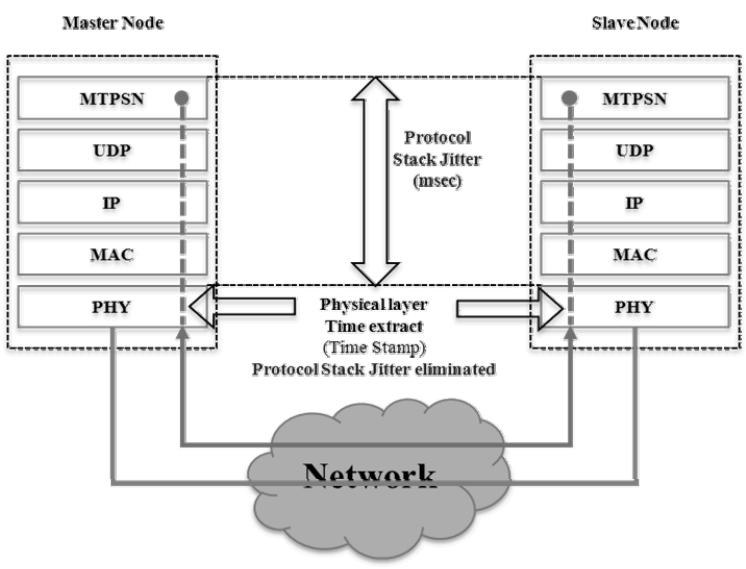

Fig. 6. Time stamping in physical layer

\subsection{Hardware Structure and Communication Method}

Fig. 7 shows the hardware structure of the proposed time-synchronization system. As seen in Fig. 7, the proposed time-synchronization system forms a clock generation class (including the GPS receiver, CPU, FPGA, $\mathrm{RF}$ frontend, DAC, ADC, Ethernet, and others).

The wireless data link realizes (and utilizes) the

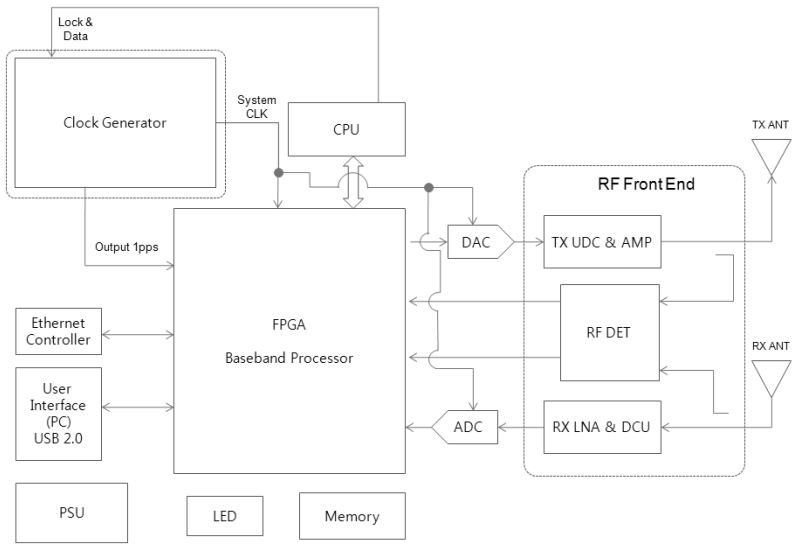

Fig. 7. Hardware structure

software defined radio (SDR). The reason for using an $\mathrm{RF}$ transceiver in the SDR form is that it is difficult both for the $\mathrm{RF}$ transceiver to control the timing at the hardware level and to avoid the delay change.

When using the proposed SDR-based RF transceiver, time-stamping can be performed, and minimization of the delay change in the two-way data link is possible. The wireless modem is used for the FPGA and the part that is not is processed in the high-speed CPU (or DSP) is processed at the software level; while the timesynchronization system supports the Ethernet outside the wireless data link and makes time-synchronization possible using the IEEE1588 PTP protocol.

For convenience, the CPU used Texas Instruments' DSP processor, which has a high-speed DSP core and an ARM RISC core and makes high-speed signal processing easy, as well as provides TCP/IP protocol stack support.

\subsection{RF Frontend}

Fig. 8 shows the detailed block diagram of the module components.

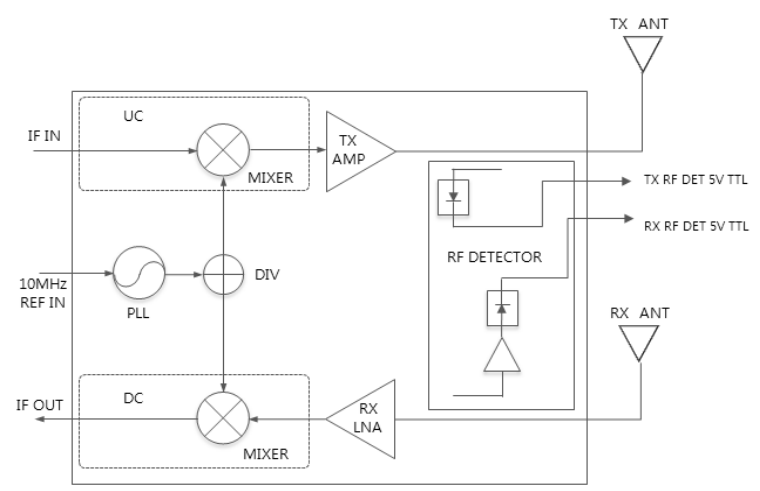

Fig. 8. RF frontend module

3.5 Time-Synchronization Protocol and Error Correction

Fig. 9 shows the time-synchronization protocol MTPSN 
movements used in the proposed time-synchronization system. The MTPSN improves the TPSN protocol, and the time-stamp message number needed in timesynchronization has been reduced sharply compared to the TPSN. The MTPSN can reduce the wireless data link burden and shorten the time required for time synchronization. Accordingly, the smaller burden and the shorter time reduce related errors in time synchronization. In Fig. 10, $\Delta \mathrm{T}$ refers to the clock difference (offset) between the master and the slave node.

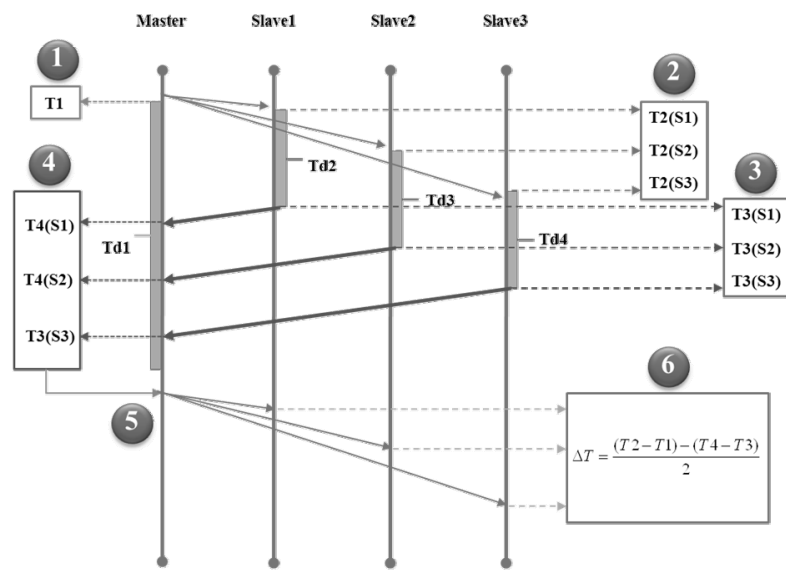

Fig. 9. Synchronization between nodes using the MTPSN

The movement order of the TPSN progresses following the order of the numbers in Fig. 9, which are as follows:

- The TPSN sends the broadcasting SyncInt message for initial synchronization

- The TPSN stores T2 time information after receiving the SyncInt message regarding time-synchronization infrastructure on the wireless network

- After sending the SyncPulse message for each slave step-by-step, the TPSN stores the T3 time information

- When not receiving the three SyncPulses during Td1 time, the TPSN requests retransmission

- SyncAck message broadcasting including T1 and T4 time information

- Correction of $\Delta \mathrm{T}$ using T1, T2, T3, and T4 after receiving the SyncAck message

In reference broadcast synchronization (RBS) [4] or timing-sync protocol for sensor networks (TPSN) [5], this method might correct the offset between clocks. Contrarily, the crystal oscillator continues to move without moving through the same frequency because of environmental factors such as temperature and humidity. The offset correction is good at ensuring short-term stability, but has disadvantages in maintaining long-term stability. Skew correction maintains the long-term stability. After making the two corrections, we can consider the corrected offset value as the new input of the skew correction, and we can estimate the skew value by minimizing the estimation error using the recursive least squares method and Kalman filter[13].

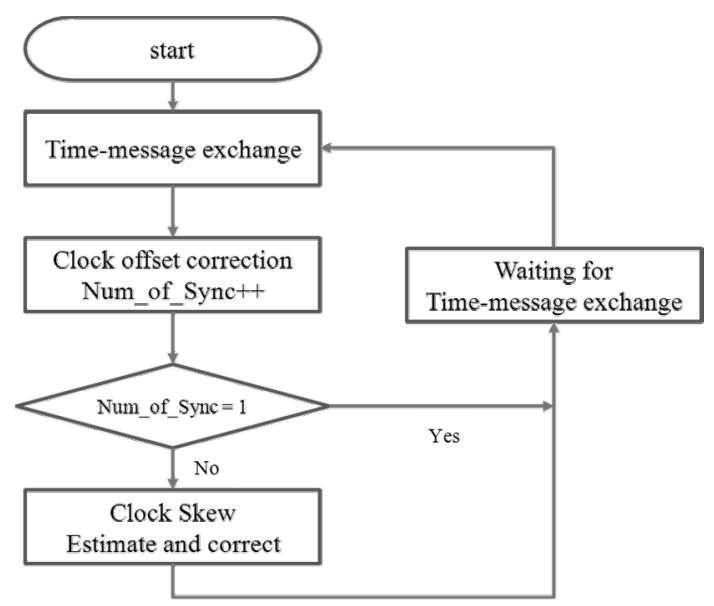

Fig. 10. Offset and skew estimation flow chart

In Fig. 10, we see the process for estimating the clock offset and skew. In the proposed system (with the clock offset and skew Gaussian distribution), the timesynchronization error is corrected using the Kalman filter.

\section{Evaluation}

The proposed system has a time--synchronization error of less than 5 ns. We have assumed that the system has a time-synchronization error of $5 \mathrm{~ns}, 10 \mathrm{~ns}, 15 \mathrm{~ns}$, and 20 ns and have applied the TDoA algorithm. After comparing the estimated results, we evaluated the performance on the basis of these results.

\subsection{TDoA Algorithm}

TDoA uses the difference in the time taken by the RF transmitter's signal to reach the $\mathrm{RF}$ receivers (separated in space). First, we measure the TDoA value of the RF receiver pair at two different locations. Using the $R F$ transmitter's location (known in advance) and the measured TDoA value, we have all the information necessary to perform the location estimation using TDoA.

\section{1) 2-Dimensional TDoA}

Time synchronization must be achieved between the receivers (BS).

There are three TDoA values TBS1, TBS2, and TBS3. 
The two-pair curve is the users' terminal location; it is used to estimate the location in two dimensions, and three BSs are needed, as shown in Fig. 11.

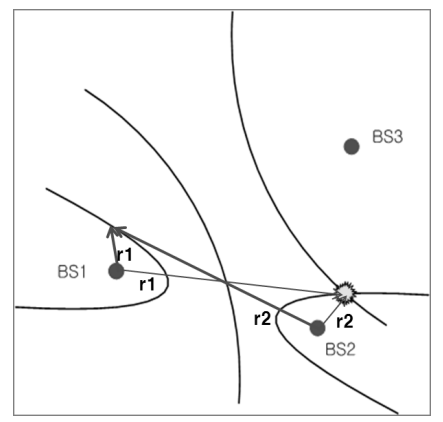

Fig. 11. Wireless location detection using TDoA in two dimensions

The TDoA $(\Delta t 12)$ is expressed in (2). As a result, the TDoA can be calculated as follows, in (3):

$$
\begin{aligned}
& \Delta t_{12}=\frac{r_{1}}{c}-\frac{r_{2}}{c} \\
& r_{1}-r_{2}=c \Delta t_{12}
\end{aligned}
$$

\section{2) 3-Dimensional TDoA}

The target location with a common TDoA value is all points on the two leaf-like curved surfaces. Threedimensional localization is possible using more than three TDoA values when the target (transmitter) is on the earth's surface, as seen in Fig. 12.
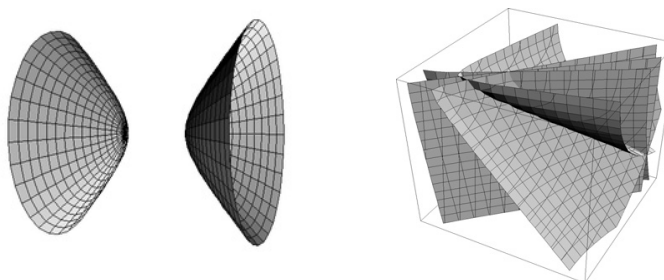

Fig. 12. Wireless location detection using TDoA in three dimensions

\section{3) Simulate TDoA-based Location Estimation}

As Fig. 1 shows, when there is a transmitter or receiver in the coordinates, the system estimates the transmitter's location. Based on the formula below, it looks for each electric wave reaching time and calculates the next. Through these calculations, three of the two leaf-like curved surfaces are selected, and the transmitter location is determined.
- Each EM wave reaching time

$$
\begin{aligned}
& T_{L}=\frac{1}{c}\left(\sqrt{\left(x-x_{L}\right)^{2}+\left(y-y_{L}\right)^{2}+\left(z-z_{L}\right)^{2}}\right. \\
& T_{C}=\frac{1}{c}\left(\sqrt{\left(x-x_{C}\right)^{2}+\left(y-y_{C}\right)^{2}+\left(z-z_{C}\right)^{2}}\right. \\
& T_{R}=\frac{1}{c}\left(\sqrt{\left(x-x_{R}\right)^{2}+\left(y-y_{R}\right)^{2}+\left(z-z_{R}\right)^{2}}\right. \\
& T_{Q}=\frac{1}{c}\left(\sqrt{\left(x-x_{Q}\right)^{2}+\left(y-y_{Q}\right)^{2}+\left(z-z_{Q}\right)^{2}}\right.
\end{aligned}
$$

- TDoA (C coordinates starting point)

$$
\begin{aligned}
& \Gamma_{L}=T_{L}-T_{C}=\frac{1}{c}\left(\sqrt{\left(x-x_{L}\right)^{2}+\left(y-y_{L}\right)^{2}+\left(z-z_{L}\right)^{2}}-\sqrt{x^{2}+y^{2}+z^{2}}\right. \\
& \Gamma_{R}=T_{R}-T_{C}=\frac{1}{c}\left(\sqrt{\left(x-x_{R}\right)^{2}+\left(y-y_{R}\right)^{2}+\left(z-z_{R}\right)^{2}}-\sqrt{x^{2}+y^{2}+z^{2}}\right. \\
& \Gamma Q=T_{Q}-T_{C}=\frac{1}{c}\left(\sqrt{\left(x-x_{Q}\right)^{2}+\left(y-y_{Q}\right)^{2}+\left(z-z_{Q}\right)^{2}}-\sqrt{x^{2}+y^{2}+z^{2}}\right.
\end{aligned}
$$

- Formulas of three of the two leaf-like curved surfaces:

$$
\begin{aligned}
& 0=A_{1} x+B_{1} y+C_{1} z+D_{1} \\
& 0=A_{2} x+B_{2} y+C_{2} z+D_{2} \\
& 0=A_{3} x+B_{3} y+C 3 z+D_{3}
\end{aligned}
$$

\section{4) Location Estimation}

Assuming there are four receivers, the system acquires a total of six TDoA values, as shown in Fig. 13. Because of the measurement error and because the six-pair curved surfaces do not have an intersection point, optimization is needed to minimize the location error [14]. When performing the location estimation using the minimum square error (MSE), the number of constraints (pair curved surfaces equation) is large, and the location error is small.

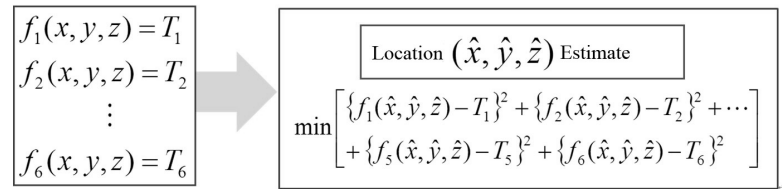

Fig. 13. Location estimation

\subsection{Location Estimation following the Receiver Arrangement Distance}

For different electronic detection distances between 10 $\mathrm{km}$ and $50 \mathrm{~km}$ with $10 \mathrm{~km}$ increments, as shown in Fig. 14, we have compared the result using the simulation. To minimize the location error in the optimization step, we perform the location estimation using the MSE. The $x$, y', z' values derived from the distance difference between the above location estimation and the location of actual target, we determined the location error. In the case of a location error drawn from the actual target standard and the location estimation from the MSE, we performed the operation 100 times each. 


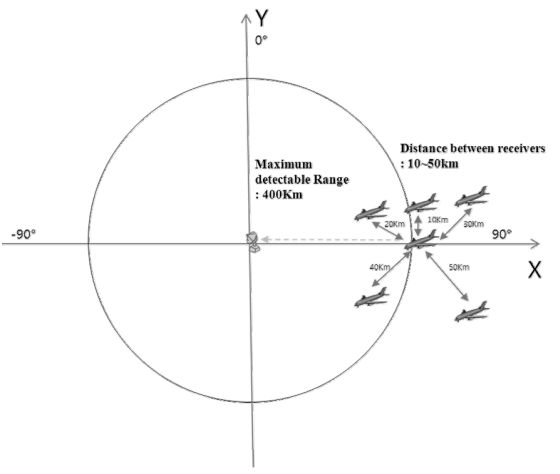

Fig. 14. Change in arrangement between receivers

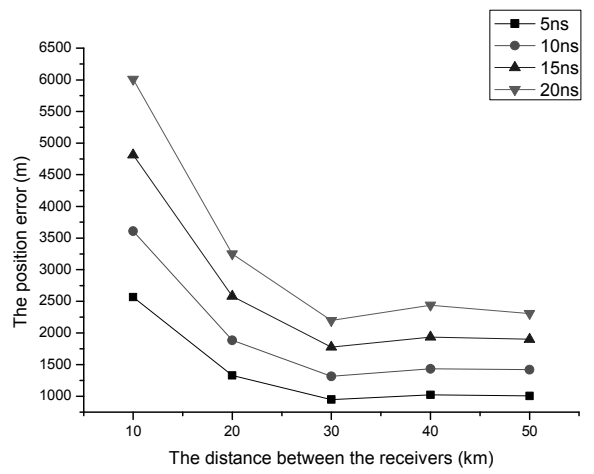

Fig. 15. Position error (receiver to receiver)

The time-synchronization error in the 5 ns case has an average error of $1865 \mathrm{~m}$, much less than that for the 20 ns case. When the electronic detection distance is large, we can derive accurate location information. When the electronic detection distance is large, the electronic detectors are known to be distributed on an open surface. As the difference between the actual target and the electronic detector increases, the area forming the pair curve decreases. A small area means the location range is smaller, compared to the large area when the distance difference that occurs is small. Based on this fact, the TDoA basis location estimation is achieved. The results are shown above.

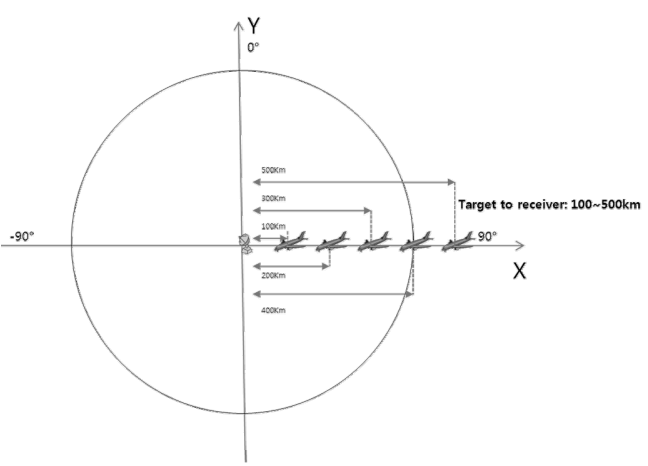

Fig. 16. Change in location between target and receiver

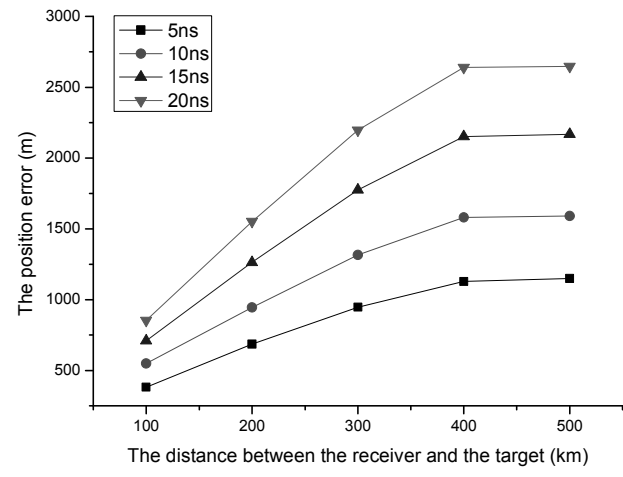

Fig. 17. Position error (target to receiver)

The distance between the receiver and the target affects the position error. As mentioned above, when the distance difference between the actual target and the electronic detector is small, the area forming the pair curve is small. Fig. 16 shows the simulation environment. Fig. 17 shows the simulation result for this environment.

\subsection{Location Estimation following the Receiver's Orbit}

In Fig. 14, we assume the distances between the receivers are $30 \mathrm{~km}$. As the receiver group moves along the circumference of a circle, we record how the average location estimation value is affected and how it progresses over four orbits, as shown below:
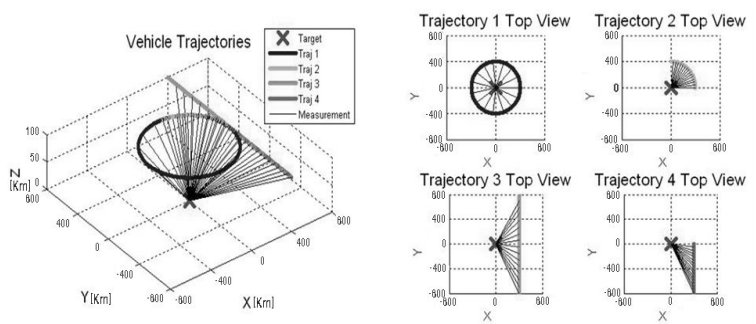

Fig. 18. Orbit of the receivers

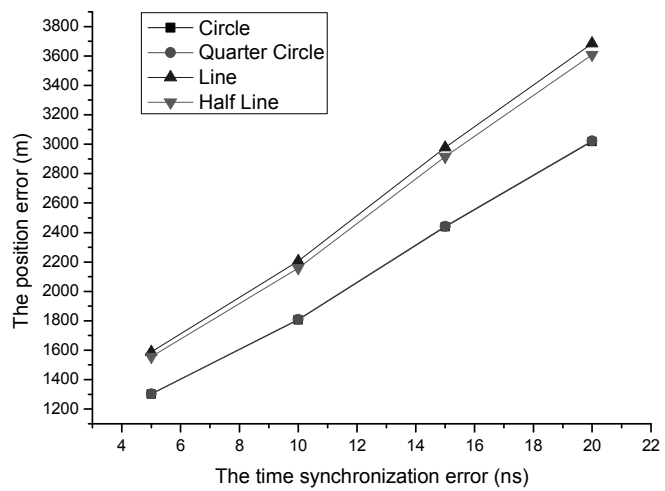

Fig. 19. Position error for the orbits 


\section{Conclusion}

In this paper, we have applied a hardware and compensation algorithm to reduce the timesynchronization error. Based on this method, we have presented a system that has a time--synchronization error that is less than 5 ns. The proposed system with a time-synchronization error less than 5 ns has a minimum error of $1250 \mathrm{~m}$, and a maximum error of $3441 \mathrm{~m}$ (an average $1867 \mathrm{~m}$ error), much less than that of the $20 \mathrm{~ns}$ system. Thus, the location estimation system performance has increased by $24.2 \%$ on an average.

In future, for the proposed system, we are planning to reduce the error to within 1 ns using the timesynchronization error correction algorithm and a highly accurate OCXO. Moreover, by creating the actual system connecting TDoA and FDoA, we will improve the location estimation system performance.

\section{Reference}

[1] Elson, J., \& Romer, K. (2002). Wireless sensor networks: A new regime for time synchronization. Proceedings of the First Workshop on Hot Topics In Networks, Princeton, New Jersey.

[2] Ganeriwal, S., Kumar, R., \& Srivastava, M. B. (2003). Timing-sync protocol for sensor networks. ACM SenSys.

[3] Sichitiu, M. L., \& Veerarittiphan, C. (2003). Simple, accurate time synchronization for wireless sensor networks. Proceeding of the IEEE Wireless Communications and Networking Conference (WCNC), New Orleans, LA.

[4] Elson, J., Girod, L. \& Estrin, D. (2002). Fine-grained network time synchronization using reference broadcasts. Proceedings of the Fifth Symposium on Operating Systems Design and Implementation (OSDI 2002), Boston, MA.

[5] Ganeriwal, S., \& Srivastava, M. (2003). Timing-sync protocol for sensor networks. ACM SenSys.

[6] Cho, H. T., Jang, H. S., \& Baek, Y. J. (2010). Practical localization system for consumer devices using zigbee networks. IEEE Trans. Consumer Electronics, 56(3), pp. 696-704

[7] Manodham T., Loyola L., \& Miki T. (2008). A NovelWireless Positioning System for Seamless Internet Connectivity based on the WLAN Infrastructure. Spriger, Wireless Pers Commun, 44(3), pp.295-309.

[8] Yunck, T. P., Melbourne, W. G., \& Thoenton, C. L. (1985). GPS-based satellite tracking system for precise positioning. IEEE Trans. Consumer Electronics, GE-23(4), pp.696-704.

[9] Ganeriwal, S., Tsigkogiannis, I., \& Shim, H. (2009). Estimating clock uncertainty for efficient duty-cycling in sensor networks. IEEE/ACM Transactions on Networking (TON), 17(3), pp.843-856.

[10] Ai, B., Shen, Y., Zhong, Z. D., \& Zhang, B. H. (2011). Enhanced sampling clock offset correction based on time domain estimation scheme. IEEE Trans. Consumer Electronics, 57(2), pp.696-704, Apr., 2011.

[11] Maggs, M.K., O'Keefe, S.G. \& Thiel, D.V. (2012). Consensus Clock Synchronization for Wireless Sensor Networks. IEEE Sensors Journal, 12(6), pp.2269-2277.

[12] Ahmad, A., Zennaro, D., Serpedin, E. \& Vangelista, L. (2012). A Factor Graph Approach to Clock Offset Estimation in Wireless Sensor Networks. IEEE Trans. Information Theory, 58(7), pp.4244-4260.

[13] Kalman, R. E. (1960) A new approach to linear filtering and prediction problems. Tran. of ASME, Journal of Basic Engineering, 82D(3), pp.34-45.

[14] Han, J. H., \& Jeong, D. K. (2002). Comparisons of error characteristics between TOA and TDoA positioning. IEEE Trans. Aerospace and Electronic Systems, 38(1), pp.307-311.

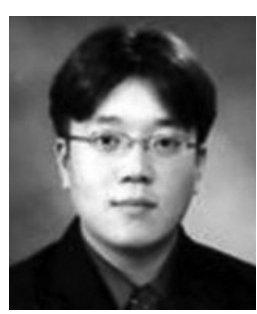

\section{Kim Jae Wan}

e-mail : jaewan.kim78@gmail.com $\mathrm{He}$ is a Ph.D. candidate in the School of Electronics and Computer Engineering at Korea University. He received a B.S. degree in Electronics and Electrical Engineering and an M.S. degree in Electronics and Computer Engineering from Korea University, Korea. His research interests include embedded systems, wireless sensor networks, mobile ad-hoc networks, vehicular ad-hoc networks, and ubiquitous computing.

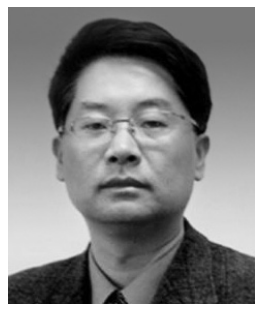

\section{Eom Doo Seop}

e-mail : eomds@korea.ac.kr

$\mathrm{He}$ is currently a Professor in the School of Electrical Engineering at Korea University since 2000. He received B.S. and M.S. degrees in Electronics Engineering from Korea University, Seoul, Korea in 1987 and 1989, respectively. In 1999, he received the Ph.D. degree in Information and Computer Sciences from Osaka University, Osaka, Japan. He joined the Communication Systems Division, Electronics and Telecommunications Research Institute (ETRI), Korea, in 1989. From September 1999 to August 2000, he was an Associate Professor at Wonkwang University, Korea. Since September 2000, he has been a Professor in the School of Electronics Engineering at Korea University. His research interests include; Communication Network Design, Wireless Sensor Networks, embedded system, RFID and Internet QoS. 